\title{
Some Operations over Interval-Valued Fuzzy Matrices
}

\author{
I. Silambarasan
}

Department of Mathematics, Annamalai University, Annamalainagar-608002, India.

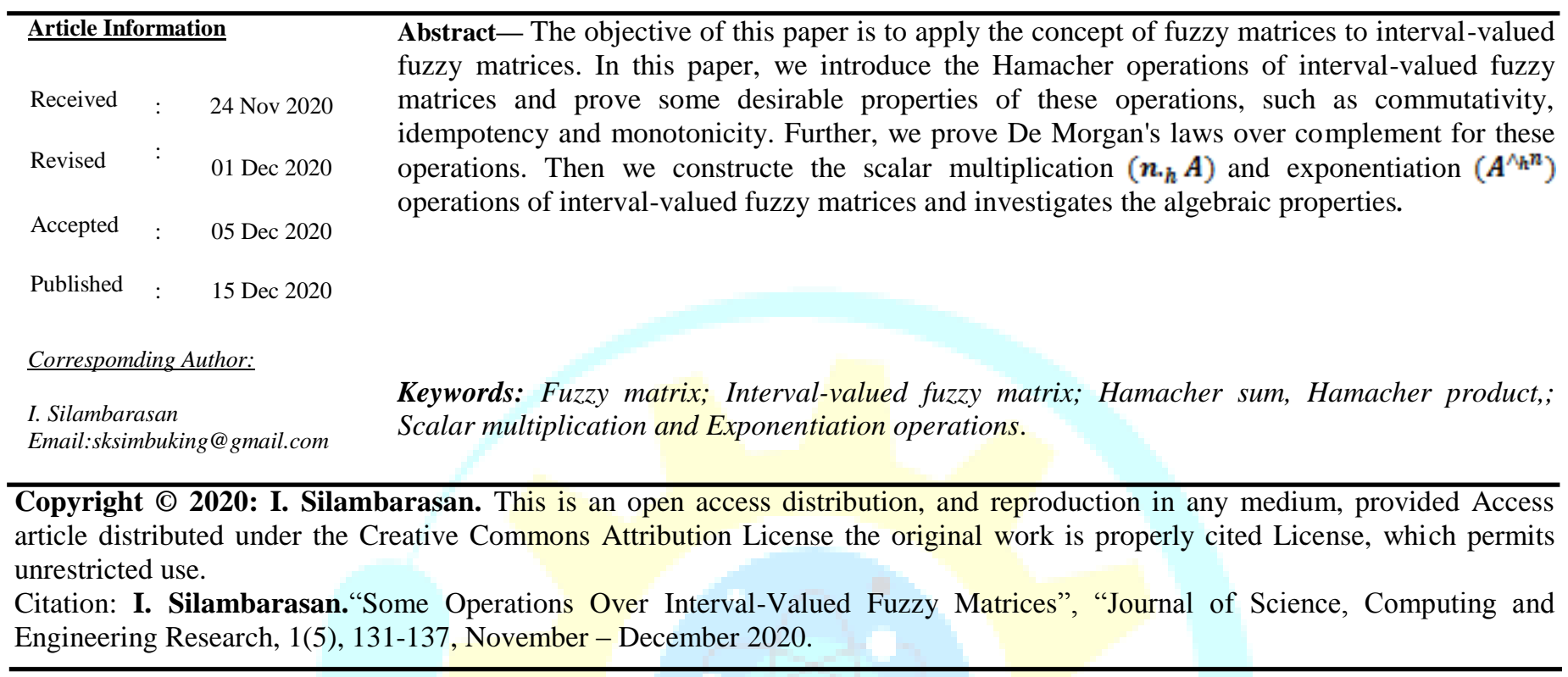

\section{INTRODUCTION}

Fuzzy matrices arise in many applications, one of which is as adjacency matrices of fuzzy relations and fuzzy relational equations have important applications in pattern classification and in handing fuzziness in knowledge based systems. Several authors presented a number of results on fuzzy matrices. In [14] Thomason initiated the study on convergence of powers of fuzzy matrix. The theory of fuzzy matrix was developed by Kim and Roush [2] as an extension of Boolean matrix, with max-min operation over the fuzzy algebra. Ragab and Emam [7] presented some properties on determinant and adjoint of a square fuzzy matrix. Ragab and Emam [8] presented some properties of the min-max composition of fuzzy matrices. We deal with interval valued fuzzy matrices (IVFM) that is, matrices whose entries are intervals and all the intervals are subintervals of the interval $[0,1]$.

Recently the concept of IVFM a generalization of fuzzy matrix was introduced and developed by shymal and pal [9], by extending the max. min operations on fuzzy algebra $\mathrm{F}=[0,1]$, for elements, $a, b \in F, a+b=\max (a, b)$ and $a-b=\min (a, b)$. In ]3], Meenakshi and Kaliraja have represented and IVFM as an interval matrix of its lower and upper limit fuzzy matrices. Anita and Pal [6] introduced intervalvalued fuzzy matrices (IVFMs) as the generalization of interval-valued fuzzy sets. Pal [5] a new type of interval-valued fuzzy matrices (IVFMs) are called interval-valued fuzzy matrices with interval-valued fuzzy rows and columns (IVFMFRCs) are defined and several important properties are investigated. Hamacher [1] proposed a more generalized t-norm and t-conorm. Hamacher operation includes the Hamacher product and
Hamacher sum. In [10], we defined two new operations called Hamacher sum and Hamacher product of fuzzy matrices and investigate the algebraic properties. This paper we extend Hamacher operations of interval-valued fuzzy matrices.

The paper is organized in three sections. In section 2, the definitions and operations on interval-valued fuzzy matrices and Hamacher operations of fuzzy matrices. In section 3 , we introduce the Hamacher operations of interval-valued fuzzy matrices and focusing on its properties. The De Morgan's law for the Hamacher operations are established in section 4. In section 5, we constructed Hamacher scalar multiplication $\left(n_{s_{h}} A\right)$ and Hamacher exponentiation $\left(A^{A_{h} n}\right)$ operations of interval-valued fuzzy matrix $A$ and investigated their algebraic properties. Section 6 concludes the paper with some future directions.

\section{PRILIMINARIES}

In this section, some basic concepts of interval-valued fuzzy matrix and Hamacher operations of fuzzy matrix is given below.

\section{Definition 2.1. [9]}

An interval-valued fuzzy matrix of order $m \times n$ is defined as, $A=\left(a_{i j j}\right)_{m \times n}$, where $a_{i j}=\left[a_{i j L}, a_{i j U}\right]$ is the $i j^{t h}$ elements of $A$, represents the membership value. All the elements of an IVFM are intervals and all the intervals are the subintervals of the interval $[0,1]$.

In the IVFM, the elements are the membership grade of some attributes, they not crisp number, so naturally some 
new operations are needed to handel such matrices. Before applying binary or unary operations between IVFMs we need clear idea about the similar operations between the elements.

\section{Definition 2.2. [9]}

An IVFM is said to be a null IVFM if all its elements are zero, i.e., all elements are $[0,0]$ and the matrix is denoted by O.

\section{Definition 2.3. [9]}

An IVFM $A=\left(a_{i j}\right)$ of order $n \times n$ is called unit IVFM or identity IVFM if all the diagonal entries of $A$ are $[1,1]$ and all other entries are $[0,0]$. It is denoted by $I_{n}$.

\section{Definition 2.4. [9]}

For any two IVFMs $A=\left(a_{i j L}, a_{i j U}\right)$ and $B=\left(b_{i j L}, b_{i j U}\right)$ of order $m \times n$ in the following.

(i) $A \vee B=\left(\max \left\{a_{i j L}, b_{i j L}\right\}, \max \left\{a_{i j U}, b_{i j U}\right\}\right)$

(ii) $A \wedge B=\left(\min \left\{a_{i j L}, b_{i j L}\right\}, \min \left\{a_{i j U}, b_{i j V}\right\}\right)$

(iii) $A^{C}=\left[1-a_{i j U}, 1-a_{i j L}\right]$

(iv) $A^{T}=\left(a_{j L}, a_{j U}\right)($ the transpose of $A)$

(v) $A \leq B$ if and only if $a_{i j L} \leq b_{i j L}$ and $a_{i j V} \leq b_{i j U}$ for all $\mathrm{i}, \mathrm{j}$.

\section{Definition 2.5. [10]}

For any fuzzy matrices $A$ and $B$ of the same size, The Hamacher sum of $A$ and $B$ is defined by $A \oplus_{H} B=\left(\frac{a_{i j}+b_{i j}-2 a_{i j} b_{i j}}{1-a_{i j} b_{i j}}\right)$. The Hamacher product of $A$ and $B$ is defined by $A \bigotimes_{H} B=\left(\frac{a_{i j} b_{i j}}{a_{i j}+b_{i j}-a_{i j} b_{i j}}\right)$.

Lemma 2.6. [10]

For $a n y, b \in[0,1] \frac{a b}{a+b-a b} \leq \frac{a+b-2 a b}{1-a b}$.

\section{SOME OPERATIONS OVER IVFMS}

In this section, based on the Definition 2.5, we define some operations for any two IVFMs $A=\left(a_{i j L}, a_{i j U}\right)$ and $B=\left(b_{i j L}, b_{i j V}\right)$ of order $m \times n$ in the following.

Definition 3.1. Let $A=\left(a_{i j L}, a_{i j V}\right)$ and $B=\left(b_{i j L}, b_{i j V}\right)$ of same size, then the Hamacher operations of interval-valued fuzzy matrices are defined as follows:

(i) The Hamacher sum of $A$ and $B$ is defined by

$A \oplus_{H} B=\left(c_{i j L}, c_{i j U}\right)$,

where $\left(c_{i j L}, c_{i j U}\right)$

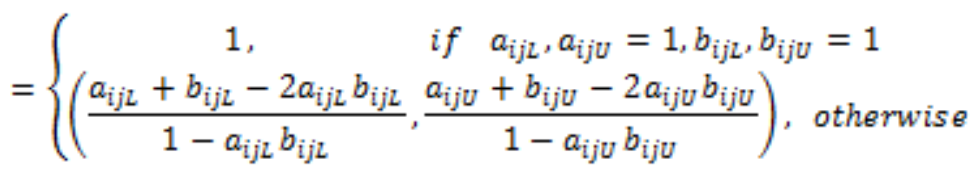

(ii) The Hamacher product of $A$ and $B$ is defined by $A \bigotimes_{H} B=\left(c_{i j L}, c_{i j U}\right) x$

where $\left(c_{i j L}, c_{i j V}\right)$

$$
=\left\{\begin{array}{cc}
0_{s} & \text { if } a_{i j L}, a_{i j U}=0, b_{i j L}, b_{i j U}=0 \\
\left(\frac{a_{i j L} b_{i j L}}{a_{i j L}+b_{i j L}-a_{i j L} b_{i j L}}, \frac{a_{i j U} b_{i j U}}{a_{i j U}+b_{i j U}-a_{i j U} b_{i j U}}\right), \text { otherwise }
\end{array}\right.
$$

respectively.

We present some basic properties of IVFMs. The commutative, associative and identity laws are valid for IVFMs under the operations addition $\oplus_{H}$ and multiplication $\otimes_{H}$. Let $F_{\operatorname{mn}}$ denote the set of all IVFMs.

Property 3.2. For any IVFMs, $A=\left(a_{i j L}, a_{i j U}\right)$ and $B=\left(b_{i j L}, b_{i j U}\right)$ of same size, then $A \Theta_{H} B \leq A \oplus_{H} B$.

Proof. By using Lemma 2.6,

$$
\begin{aligned}
& \left(\frac{a_{i j L} b_{i j L}}{a_{i j L}+b_{i j L}-a_{i j L} b_{i j L}} \leq \frac{a_{i j L}+b_{i j L}-2 a_{i j L} b_{i j L}}{1-a_{i j L} b_{i j L}}\right) \\
& \left(\frac{a_{i j U} b_{i j U}}{a_{i j U}+b_{i j U}-a_{i j U} b_{i j U}} \leq \frac{a_{i j U}+b_{i j U}-2 a_{i j U} b_{i j U}}{1-a_{i j U} b_{i j U}}\right)
\end{aligned}
$$
for all $\mathrm{i}, \mathrm{j}$.

Hence, $A \Theta_{H} B \leq A \oplus_{H} B$.

Property 3.3. For any IVFM $A$,

$$
\text { (i) } A \oplus_{H} A \geq A \text {, }
$$$$
\text { (ii) } A \otimes_{H} A \leq A \text {. }
$$

\section{Proof}

. (i) $A \oplus_{H} A=\left(\frac{2 a_{i j L}-2 a_{i j L}^{2}}{1-a_{i j L}^{2}}, \frac{2 a_{i j U}-2 a_{i j U}^{2}}{1-a_{i j U}^{2}}\right)$

$=\left(\frac{2 a_{i j L}}{1+a_{i j L}}, \frac{2 a_{i j U}}{1+a_{i j U}}\right) \geq\left(a_{i j L}, a_{i j U}\right)=A$.

Since $a_{i j L} \leq \frac{2 a_{i j L}}{1+a_{i j L}}, a_{i j U} \leq \frac{2 a_{i j U}}{1+a_{i j U}}$ for all i,j.

Therefore $A \oplus_{H} A \geq A$.

$$
\begin{aligned}
& \text { (ii) } A \bigotimes_{H} A=\left(\frac{a_{i j L}^{2}}{2 a_{i j L}-a_{i j L}^{2}}, \frac{a_{i j U}^{2}}{2 a_{i j U}-a_{i j U}^{2}}\right) \\
& =\left(\frac{a_{i j L}}{2-a_{i j L}}, \frac{a_{i j U}}{2-a_{i j U}}\right) \leq\left(a_{i j L}, a_{i j U}\right)=A
\end{aligned}
$$

for all i,j. Therefore $A \bigotimes_{H} A \leq A$. 


\section{Some Operations Over Interval-Valued Fuzzy Matrices}

Available at https://jscer.org

The operations $\oplus_{H}$ and $\bigotimes_{H}$ are commutative as well as associative and identity and with respect to $\bigoplus_{H}$ and $\otimes_{H}$ are exist.

Property 3.4 For any IVFMs, $A=\left(a_{i j L}, a_{i j J}\right)$, $B=\left(b_{i j L}, b_{i j U}\right)$ and $C=\left(c_{i j L}, c_{i j U}\right)$ of same size, then

(i) $A \oplus_{H} B=B \oplus_{H} A$,

(ii) $\left(A \oplus_{H} B\right) \oplus_{H} C=A \oplus_{H}\left(B \oplus_{H} C\right)$,

(iii) $A \otimes_{H} B=B \otimes_{H} A$,

$(i v)\left(A \otimes_{H} B\right) \otimes_{H} C=A \otimes_{H}\left(B \otimes_{H} C\right)$.

Proof. We prove (i) and (iii), other can be proved analogously.

(i) $A \oplus_{H} B=\left(\frac{a_{i j L}+b_{i j L}-2 a_{i j L} b_{i j L}}{1-a_{i j L} b_{i j L}}, \frac{a_{i j U}+b_{i j U}-2 a_{i j U} b_{i j U}}{1-a_{i j U} b_{i j U}}\right)$ $=\left(\frac{b_{i j L}+a_{i j L}-2 b_{i j L} a_{i j L}}{1-b_{i j L} a_{i j L}}, \frac{b_{i j U}+a_{i j U}-2 b_{i j U} a_{i j U}}{1-b_{i j U} a_{i j U}}\right)$ $=B \oplus_{H} A$.

(iii) $A \otimes_{H} B=\left(\frac{a_{i j L} b_{i j L}}{a_{i j L}+b_{i j L}-a_{i j L} b_{i j L}}, \frac{a_{i j U} b_{i j U}}{a_{i j U}+b_{i j U}-a_{i j U} b_{i j U}}\right)$

$$
\begin{aligned}
& =\left(\frac{b_{i j L} a_{i j L}}{b_{i j L}+a_{i j L}-b_{i j L} a_{i j L}} \cdot \frac{b_{i j U} a_{i j U}}{b_{i j U}+a_{i j U}-b_{i j U} a_{i j U}}\right) \\
& =B \otimes_{H} A
\end{aligned}
$$

The following properties are obvious.

Property 3.5 For any IVFMs, $A=\left(a_{i j L}, a_{i j U}\right)$ and $B=\left(b_{i j L}, b_{i j U}\right)$ of same size, then

(i) $A \oplus_{H} B \geq(A \vee B)$,

(ii) $A \otimes_{H} B \leq(A \vee B)$,

(iii) $A \oplus_{H} B \geq(A \wedge B)$,

(ii) $A \otimes_{H} B \leq(A \wedge B)$.

Property 3.6 For any IVFM $A$,

(i) $A \oplus_{H} O=O \oplus_{H} A=A$,

(ii) $A \otimes_{H} J=J \otimes_{H} A=A$,

(iii) $A \oplus_{H} J=J$,

(iv) $A \otimes_{H} O=O$.
Proof. We prove (i) and (ii), other can be proved analogously.

(i) $A \oplus_{H} O=\left(\frac{a_{i j L}+0-2 a_{i j L} \cdot 0}{1-a_{i j L} \cdot 0}, \frac{a_{i j U}+0-2 a_{i j U} \cdot 0}{1-a_{i j U} \cdot 0}\right)$

$$
=\left(a_{i j L}, a_{i j U}\right)=A \text {. }
$$

$O \oplus_{H} A=\left(\frac{0+a_{i j L}-2 \cdot 0 \cdot a_{i j L}}{1-0 \cdot a_{i j L}}, \frac{0+a_{i j U}-2.0 \cdot a_{i j U}}{1-0 \cdot a_{i j U}}\right)$

$$
=\left(a_{i j L}, a_{i j U}\right)=A \text {. }
$$

(ii) $A \otimes_{H} J=\left(\frac{a_{i j L} \cdot 1}{a_{i j L}+1-a_{i j L} \cdot 1}, \frac{a_{i j U} \cdot 1}{a_{i j U}+1-a_{i j U} \cdot 1}\right)$

$$
=\left(a_{i j L}, a_{i j U}\right)=A \text {. }
$$

$$
\begin{aligned}
J \otimes_{H} A & =\left(\frac{1 \cdot a_{i j L}}{1+a_{i j L}-1 \cdot a_{i j L}}, \frac{1 \cdot a_{i j U}}{1+a_{i j U}-1 \cdot a_{i j U}}\right) \\
& =\left(a_{i j L}, a_{i j U}\right)=A .
\end{aligned}
$$

Thus $\left(F_{m n}, \oplus_{H}\right)$ and $\left(F_{\operatorname{mn}}, \Theta_{H}\right)$ forms a commutative monoid.

The operators $\bigoplus_{H}$ and $\otimes_{H}$ do not obey the De Morgan's laws over transpose.

Property 3.7 For any IVFMs, $A=\left(a_{i j L}, a_{i j U}\right)$ and $B=\left(b_{i j L}, b_{i j U}\right)$ of same size, then

(i) $\left(A \oplus_{H} B\right)^{T}=A^{T} \oplus_{H} B^{T}$,

(ii) $\left(A \otimes_{H} B\right)^{T}=A^{T} \otimes_{H} B^{T} . \quad$ Where $\quad A^{T} \quad$ is the transpose of $A$.

Property 3.8 For any IVFMs, $A=\left(a_{i j L}, a_{i j U}\right)$ and $B=\left(b_{i j L}, b_{i j U}\right)$ of same size, if $A \leq B$, then $A \otimes_{H} C \leq B \otimes_{H} C$

Proof. Let $a_{i j L} \leq b_{i j L}$ and $a_{i j U} \leq b_{i j U}$ for all i,j.

$a_{i j L} c_{i j L}^{2} \leq b_{i j L} c_{i j L}^{2}$

$a_{i j L} c_{i j L}^{2}+a_{i j L} b_{i j L} c_{i j L}\left(1-c_{i j L}\right) \leq b_{i j L} c_{i j L}^{2}+a_{i j L} b_{i j L} c_{i j L}\left(1-c_{i j L}\right)$

$a_{i j L} c_{i j L}^{2}+a_{i j L} b_{i j L} c_{i j L}-a_{i j L} b_{i j L} c_{i j L}^{2} \leq b_{i j L} c_{i j L}^{2}+a_{i j L} b_{i j L} c_{i j L}-a_{i j L} b_{i}$

$a_{i j L} c_{i j L}\left(c_{i j L}+b_{i j L}-b_{i j L} c_{i j L}\right) \leq b_{i j L} c_{i j L}\left(c_{i j L}+a_{i j L}-a_{i j L} c_{i j L}\right)$ 


\section{Some Operations Over Interval-Valued Fuzzy Matrices}

Available at https://jscer.org

$$
\frac{a_{i j L} c_{i j L}}{a_{i j L}+c_{i j L}-a_{i j L} c_{i j L}} \leq \frac{b_{i j L} c_{i j L}}{b_{i j L}+c_{i j L}-b_{i j L} c_{i j L}}
$$

for all $\mathrm{i}, \mathrm{j}$,

and similarly we prove that,

$\frac{a_{i j U} c_{i j U}}{a_{i j U}+c_{i j U}-a_{i j U} c_{i j U}} \leq \frac{b_{i j U} c_{i j U}}{b_{i j U}+c_{i j U}-b_{i j U} c_{i j U}}$ for all $\mathrm{i}, \mathrm{j}$.

$\begin{array}{llll}\text { Property } & 3.9 & \text { For any IVFMs, }\end{array}$ $A=\left(a_{i j L}, a_{i j U}\right), B=\left(b_{i j L}, b_{i j U}\right)$ and $C=\left(c_{i j L}, c_{i j U}\right)$ of same size, if $A \leq B$, then $A \oplus_{H} C \leq B \oplus_{H} C$

Proof. Let $a_{i j L} \leq b_{i j L}$ and $a_{i j U} \leq b_{i j U}$ for all i,j.

$a_{i j L}\left(1-c_{i j L}\right)^{2} \leq b_{i j L}\left(1-c_{i j L}\right)^{2}$

$\rightarrow a_{i j L}\left(1-2 c_{i j L}+c_{i j L}^{2}\right) \leq b_{i j L}\left(1-2 c_{i j L}+c_{i j L}^{2}\right)$

$\rightarrow a_{i j L}-2 a_{i j L} c_{i j L}+a_{i j L} c_{i j L}^{2} \leq b_{i j L}-2 b_{i j L} c_{i j L}+b_{i j L} c_{i j L}^{2}$

$a_{i j L}-2 a_{i j L} c_{i j L}+a_{i j L} c_{i j L}^{2}+\overrightarrow{\left(c_{i j L}\right.}-a_{i j L} b_{i j L} c_{i j L}+$

$\left.2 a_{i j L} b_{i j L} c_{i j L}^{2}\right) \leq b_{i j L}-2 b_{i j L} c_{i j L} b_{i j L} c_{i j L}^{2}+\left(c_{i j L}-\right.$ $\left.a_{i j L} b_{i j L} c_{i j L}+2 a_{i j L} b_{i j L} c_{i j L}^{2}\right)$

$a_{i j L}+c_{i j L}-2 a_{i j L} c_{i j L}-a_{i j L} b_{i j L} c_{i j L}-b_{i j L} c_{i j L}^{2}+$ $2 a_{i j L} b_{i j L} c_{i j L}^{2} \leq b_{i j L}+c_{i j L}-2 b_{i j L} c_{i j L}-a_{i j L} b_{i j L} c_{i j L}-$ $a_{i j L} c_{i j L}^{2}+2 a_{i j L} b_{i j L} c_{i j L}^{2}$

$a_{i j L}+c_{i j L}-2 a_{i j L} c_{i j L}-b_{i j L} c_{i j L}\left(a_{i j L}+c_{i j L}-2 a_{i j L} c_{i j L}\right) \leq$ $b_{i j L}+c_{i j L}-2 b_{i j L} c_{i j L}-a_{i j L} c_{i j L}\left(b_{i j L}+c_{i j L}-2 b_{i j L} c_{i j L}\right)$

\left.${\overrightarrow{\left(a_{i j L}\right.}}+c_{i j L}-2 a_{i j L} c_{i j L}\right)\left(1-b_{i j L} c_{i j L}\right) \leq\left(b_{i j L}+c_{i j L}-\right.$ $\left.2 b_{i j L} c_{i j L}\right)\left(1-a_{i j L} c_{i j L}\right)$

$\left(\frac{a_{i j L}+c_{i j L}-2 a_{i j L} c_{i j L}}{1-a_{i j L} c_{i j L}}\right) \leq\left(\frac{b_{i j L}+c_{i j L}-2 b_{i j L} c_{i j L}}{1-b_{i j L} c_{i j L}}\right)$ for all $\mathrm{i}, \mathrm{j}$.

and similarly we prove that,

$\left(\frac{a_{i j U}+c_{i j U}-2 a_{i j U} c_{i j U}}{1-a_{i j U} c_{i j U}}\right) \leq\left(\frac{b_{i j U}+c_{i j U}-2 b_{i j U} c_{i j U}}{1-b_{i j U} c_{i j U}}\right)$

for all $\mathrm{i}, \mathrm{j}$.
Property 3.10 For any IVFMs, $A=\left(a_{i j L}, a_{i j U}\right)$ and $B=\left(b_{i j L}, b_{i j U}\right)$ of same size, then

$(i)(A \wedge B) \oplus_{H}(A \vee B)=A \oplus_{H} B$,

$(i i)(A \wedge B) \otimes_{H}(A \vee B)=A \otimes_{H} B$,

$(i)\left(A \oplus_{H} B\right) \wedge\left(A \otimes_{H} B\right)=A \otimes_{H} B$,

(ii) $\left(A \oplus_{H} B\right) \vee\left(A \otimes_{H} B\right)=A \oplus_{H} B$.

Proof. We prove (i) and (ii), other can be proved analogously.

$(i)(A \wedge B) \oplus_{H}(A \vee B)$

$=\left[\min \left(a_{i j L}, b_{i j L}\right), \min \left(a_{i j U}, b_{i j U}\right) \oplus_{H} \max \left(a_{i j L}, b_{i j L}\right), \max \left(a_{i j U}, b_{i j U}\right)\right]$

$=\left(\begin{array}{l}\frac{\left(\min \left(a_{i j L}, b_{i j L}\right)+\max \left(a_{i j L}, b_{i j L}\right)-2 \min \left(a_{i j L}, b_{i j L}\right) \max \left(a_{i j L}, b_{i j L}\right)\right)}{\left(1-\min \left(a_{i j L}, b_{i j L}\right) \max \left(a_{i j L}, b_{i j L}\right)\right)}, \\ \frac{\left(\min \left(a_{i j U}, b_{i j U}\right)+\max \left(a_{i j U}, b_{i j U}\right)-2 \min \left(a_{i j U}, b_{i j U}\right) \max \left(a_{i j U}, b_{i j U}\right)\right)}{\left(1-\min \left(a_{i j U}, b_{i j U}\right) \max \left(a_{i j U}, b_{i j U}\right)\right)}\end{array}\right)$

$=\left(\frac{a_{i j L}+b_{i j L}-2 a_{i j L} b_{i j L}}{1-a_{i j L} b_{i j L}}, \frac{a_{i j U}+b_{i j U}-2 a_{i j U} b_{i j U}}{1-a_{i j U} b_{i j U}}\right)$

for all $\mathrm{i}, \mathrm{j}$.

.$=A \oplus_{H} B$.

\section{(ii) $(A \wedge B) \otimes_{H}(A \vee B)$}

$=\left[\begin{array}{c}\min \left(a_{i j L}, b_{i j L}\right), \min \left(a_{i j U}, b_{i j U}\right) \bigotimes_{H} \\ \max \left(a_{i j L}, b_{i j L}\right), \max \left(a_{i j U}, b_{i j U}\right)\end{array}\right]$

$=\left(\begin{array}{c}\frac{\min \left(a_{i j L}, b_{i j L}\right) \max \left(a_{i j L}, b_{i j L}\right)}{\left(\min \left(a_{i j L}, b_{i j L}\right)+\max \left(a_{i j L}, b_{i j L}\right)-\min \left(a_{i j L}, b_{i j L}\right) \max \left(a_{i j L}, b_{i j L}\right)\right)}, \\ \frac{\min \left(a_{i j U}, b_{i j U}\right) \max \left(a_{i j U}, b_{i j U}\right)}{\left(\min \left(a_{i j U}, b_{i j U}\right)+\max \left(a_{i j U}, b_{i j U}\right)-\min \left(a_{i j U}, b_{i j U}\right) \max \left(a_{i j U}, b_{i j U}\right)\right)}\end{array}\right)$
$=\left(\frac{a_{i j L} b_{i j L}}{a_{i j L}+b_{i j L}-a_{i j L} b_{i j L}}, \frac{a_{i j U} b_{i j U}}{a_{i j U}+b_{i j U}-a_{i j U} b_{i j U}}\right)$ for all i,j.

$=A \otimes_{H} B$.

The following Properties are obvious.

Property 3.11 For any IVFMs,

$A=\left(a_{i j L}, a_{i j U}\right), B=\left(b_{i j L}, b_{i j U}\right)$ and $C=\left(c_{i j L}, c_{i j U}\right)$ of same size, then 


$$
\begin{aligned}
& (i) A \oplus_{H}(B \vee C)=\left(A \oplus_{H} B\right) \vee\left(A \oplus_{H} C\right), \\
& (i i) A \otimes_{H}(B \vee C)=\left(A \otimes_{H} B\right) \vee\left(A \otimes_{H} C\right), \\
& (i i i)(A \vee B) \oplus_{H} C=\left(A \oplus_{H} C\right) \vee\left(B \oplus_{H} C\right), \\
& (i v)(A \wedge B) \oplus_{H} C=\left(A \oplus_{H} C\right) \wedge\left(B \oplus_{H} C\right),
\end{aligned}
$$

Property 3.12 For any IVFMs,

$A=\left(a_{i j L}, a_{i j U}\right), B=\left(b_{i j L}, b_{i j U}\right)$ and $C=\left(c_{i j L}, c_{i j U}\right)$ of same size, then

(i) $A \oplus_{H}(B \wedge C)=\left(A \oplus_{H} B\right) \wedge\left(A \oplus_{H} C\right)$,

$(i i) A \otimes_{H}(B \wedge C)=\left(A \otimes_{H} B\right) \wedge\left(A \otimes_{H} C\right)$,

$(i i i)(A \vee B) \otimes_{H} C=\left(A \otimes_{H} C\right) \vee\left(B \otimes_{H} C\right)$,

$(i v)(A \wedge B) \otimes_{H} C=\left(A \otimes_{H} C\right) \wedge\left(B \otimes_{H} C\right)$.

\section{ON COMPLEMENT OF IVFMS}

In this section, The complement of on IVFM is used to analysis the complement nature of any system. For example, if $\mathrm{A}$ represents the crowdness of the roads at a particular time period then its complement $A^{C}$ represents the clearness of the roads. Using the following results we can study the complement nature of a system with the help of original on IVFMs. The operator complement obey the De Morgan's law for the operator $\oplus_{H}$ and $\bigotimes_{H}$. This is established in the following property.

Property 4.1 For any IVFMs, $A=\left(a_{i j L}, a_{i j U}\right)$ and $B=\left(b_{i j L}, b_{i j U}\right)$ of same size, then

(i) $\left(A \oplus_{H} B\right)^{C}=A^{C} \otimes_{H} B^{C}$,

(ii) $\left(A \otimes_{H} B\right)^{C}=A^{C} \oplus_{H} B^{C}$,

(iii) $\left(A \oplus_{H} B\right)^{C} \leq A^{C} \oplus_{H} B^{C}$,

$(i v)\left(A \otimes_{H} B\right)^{C} \geq A^{C} \otimes_{H} B^{C}$.

Proof. (i) $A^{C} \otimes_{H} B^{C}$

$$
\begin{aligned}
& =\left(\left(1-a_{i j U} 1-a_{i j L}\right) \otimes_{H}\left(1-b_{i j U} 1-b_{i j L}\right)\right) \\
& =\left(\frac{\left(1-a_{i j U}\right)\left(1-b_{i j U}\right)}{\left(1-a_{i j U}\right)+\left(1-b_{i j U}\right)-\left(1-a_{i j U}\right)\left(1-b_{i j U}\right)}\right) \\
& =\left(\frac{\left(1-b_{i j U}-a_{i j U}+a_{i j U} b_{i j U}\right)}{\left(1-a_{i j U}\right)+\left(1-b_{i j U}\right)-\left(1+b_{i j U}+a_{i j U}-a_{i j U} b_{i j U}\right)}\right) \\
& =\left(\frac{1-b_{i j U}-a_{i j U}+a_{i j U} b_{i j U}}{2-a_{i j U}-b_{i j U}-1+b_{i j U}+a_{i j U}-a_{i j U} b_{i j U}}\right)
\end{aligned}
$$

$$
\begin{aligned}
& =\left(\frac{1+a_{i j U} b_{i j U}-a_{i j U}-b_{i j U}}{1-a_{i j U} b_{i j U}}\right) \\
& =\left(\frac{\left(1-a_{i j U}\right)\left(1-b_{i j U}\right)}{1-a_{i j U} b_{i j U}}\right) \\
& =\left(1-\frac{a_{i j U}+b_{i j U}-2 a_{i j U} b_{i j U}}{1-a_{i j U} b_{i j U}}\right)
\end{aligned}
$$

for all $\mathrm{i}, \mathrm{j}$.

Similarly we prove that, $\left(1-\frac{a_{i j L}+b_{i j L}-2 a_{i j j L} b_{i j k}}{1-a_{i j L} b_{i j L}}\right)$ for all $i, j$.

$=\left(A \oplus_{H} B\right)^{C}$.

$(i i) A^{C} \oplus_{H} B^{C}=\left(\left(1-a_{i j U} 1-a_{i j L}\right) \oplus_{H}\left(1-b_{i j U^{x}} 1-b_{i j L}\right)\right)$

$=\left(\frac{\left(1-a_{i j U}\right)+\left(1-b_{i j U}\right)-2\left(1-a_{i j U}\right)\left(1-b_{i j U}\right)}{1-\left(1-a_{i j U}\right)\left(1-b_{i j U}\right)}\right)$

$=\left(\frac{2-a_{i j U}-b_{i j U}-2\left(1-b_{i j U}-a_{i j U}+a_{i j U} b_{i j U}\right)}{1-\left(1-b_{i j U}-a_{i j U}+a_{i j U} b_{i j U}\right)}\right)$

$=\left(\frac{2-a_{i j U}-b_{i j U}-2+2 b_{i j U}+2 a_{i j U}-2 a_{i j U} b_{i j U}}{1-1+b_{i j U}+a_{i j U}-a_{i j U} b_{i j U}}\right)$

$=\left(\frac{a_{i j U}+b_{i j U}-2 a_{i j U} b_{i j U}}{a_{i j U}+b_{i j U}-a_{i j U} b_{i j U}}\right)$

$=\left(1-\frac{a_{i j U^{b} b_{i j U}}}{a_{i j U}+b_{i j U}-a_{i j U} b_{i j U}}\right)$ for all $i, j$.

Similarly we prove that,

$\left(1-\frac{a_{i j L} b_{i j L}}{a_{i j L}+b_{i j L}-a_{i j L} b_{i j L}}\right)$ for all $i, j$.

$=\left(A \otimes_{H} B\right)^{C}$.

(iii) From Property 3.2.

$A \oplus_{H} B \geq A \otimes_{H} B$

Then $\left(A \oplus_{H} B\right)^{C} \leq\left(A \otimes_{H} B\right)^{C}=A^{C} \oplus_{H} B^{C}$.

$(i v)\left(A \otimes_{H} B\right)^{C} \geq\left(A \oplus_{H} B\right)^{C}=A^{C} \otimes_{H} B^{C}$.

\section{SCALAR MULTIPLICATION AND EXPONENTIATION OPERATIONS OF IVFMS}

We defined the following operations over Hamacher operations of IVFMs . In this section, we construct Hamacher scalar multiplication $\left(n_{\cdot_{h}} A\right)$ and Hamacher exponentiation $\left(A^{\wedge_{h} n}\right)$ operations of IVFM $A$ and investigate their algebraic properties. 
Based on the Definition 3.1, Hamacher sum and Hamacher product over two IVFMs $A$ and $B$ are further indicated as the following operations.

Theorem 5.1 If $n$ is any positive integer and $A$ is a IVFM, then the Hamacher scalar multiplication operation $\left(._{h}\right)$ is

$n_{\cdot h} A=A \oplus_{n} \underbrace{}_{n} \oplus_{h} A=\left(\frac{n a_{i j L}}{1+(n-1) a_{i j L}}, \frac{n a_{i j U}}{1+(n-1) a_{i j U}}\right)$.

Proof. Mathematical induction can be used to prove that the above equation (5.1) holds for all positive integer $n$. The equation (5.1) is called $P(n)$. Using the above Definition 3.1 of Hamacher sum (i), $A \oplus_{h} B$ we have:

$A_{\times h} A=\left(\frac{a_{i j L}+a_{i j L}-2 a_{i j L} a_{i j L}}{1-a_{i j L} a_{i j L}}, \frac{a_{i j U}+a_{i j U}-2 a_{i j U} a_{i j U}}{1-a_{i j U} a_{i j U}}\right)$

$=\left(\frac{2 a_{i j L}-2 a_{i j L}^{2}}{1-a_{i j L}^{2}}, \frac{2 a_{i j U}-2 a_{i j U}^{2}}{1-a_{i j U}^{2}}\right)$.

$=\left(\frac{2 a_{i j L}\left(1-a_{i j L}\right)}{1-a_{i j L}^{2}}, \frac{2 a_{i j U}\left(1-a_{i j U}\right)}{1-a_{i j U}^{2}}\right) x$

$=\left(\frac{2 a_{i j L}}{1+a_{i j L}}, \frac{2 a_{i j U}}{1+a_{i j U}}\right)$

$=\left(\frac{2 a_{i j L}}{1+(2-1) a_{i j L}}, \frac{2 a_{i j U}}{1+(2-1) a_{i j U}}\right)$.

since $a_{i j L}=(2-1) a_{i j L}, a_{i j U}=(2-1) a_{i j U}$.

$P(n)$ holds.

Suppose that equation (5.1) holds for $n=m$,

$i_{x} e_{x s} m_{* h} A=A \oplus_{h \ldots .} \oplus_{h} A=\left(\frac{m \cdot a_{i j L}}{1+(m-1) a_{i j L}}, \frac{m \cdot a_{i j U}}{1+(m-1) a_{i j U}}\right) a_{i j U}=(2-1) a_{i j U}$.

.Then, $\quad(m+1){ }_{\cdot h} A=\left(\left(m_{\cdot h} A\right) \oplus_{h} A\right)$

$=\left(\begin{array}{l}\frac{\frac{m \cdot a_{i j L}}{1+(m-1) a_{i j L}}+a_{i j L}-2 \frac{m \cdot a_{i j L}}{1+(m-1) a_{i j L}} \cdot a_{i j L}}{1-\frac{m \cdot a_{i j L}}{1+(m-1) a_{i j L}} \cdot a_{i j L}} \\ \frac{m \cdot a_{i j U}}{1+(m-1) a_{i j U}}+a_{i j U}-2 \frac{m \cdot a_{i j U}}{1+(m-1) a_{i j U}} \cdot a_{i j U} \\ 1-\frac{m \cdot a_{i j U}}{1+(m-1) a_{i j U}} \cdot a_{i j U}\end{array}\right)$

$=\left(\frac{a_{i j L}(m+1)\left(1-a_{i j L}\right)}{\left(1+m \cdot a_{i j L}\right)\left(1-a_{i j L}\right)}, \frac{a_{i j U}(m+1)\left(1-a_{i j U}\right)}{\left(1+m \cdot a_{i j U}\right)\left(1-a_{i j U}\right)}\right)$

$$
\begin{aligned}
& =\left(\frac{(m+1) a_{i j L}}{1+m_{*} a_{i j L}}, \frac{(m+1) a_{i j U}}{1+m_{*} a_{i j U}}\right) \\
& =\left(\frac{(m+1) a_{i j L}}{1+[(m+1)-1] a_{i j L}}, \frac{(m+1) a_{i j U}}{1+[(m+1)-1] a_{i j U}}\right) .
\end{aligned}
$$

(5.1) So, when $n=m+1$,

$$
n_{r_{h}} A=A \underset{n}{\oplus_{h} \ldots} \oplus_{h} A=\left(\frac{n a_{i j L}}{1+(n-1) a_{i j L}}, \frac{n a_{i j U}}{1+(n-1) a_{i j U}}\right)
$$

also holds.

Using the induction hypothesis that $P(n)$ holds for any positive integer $n$.

Theorem 5.2 If $n$ is any positive integer and $A$ is a IVFM, then the Hamacher exponentiation operation $\left(\wedge_{h}\right)$ is

$$
A^{A_{h}{ }^{n}}=A Q_{h} \stackrel{n}{-} \otimes_{h} A=\left(\frac{a_{i j L}}{n-(n-1) a_{i j L}}, \frac{a_{i j U}}{n-(n-1) a_{i j V}}\right)
$$

Proof. Mathematical induction can be used to prove that the above equation (5.2) holds for all positive integer $n$. The equation (5.2) is called $P(n)$. Using the above Definition 3.1 of Hamacher product (ii), $A \otimes_{h} B$ we have:

$$
\begin{aligned}
& A^{A / h^{A}}=\left(\frac{a_{i j L}^{2}}{2 a_{i j L}-a_{i j L}^{2}} \cdot \frac{a_{i j U}^{2}}{2 a_{i j L}-a_{i j U}^{2}}\right) \\
& =\left(\frac{a_{i j L}^{2}}{a_{i j L}\left(2-a_{i j L L}\right)} \frac{a_{i j U}^{2}}{a_{i j U}\left(2-a_{i j U)}\right)}\right) \\
& =\left(\frac{a_{i j L}}{2-a_{i j L}}, \frac{a_{i j J}}{2-a_{i j J}}\right) \text {. }
\end{aligned}
$$

$A^{N_{h}{ }^{n}}=\left(\frac{a_{i j L}}{n-(n-1) a_{i j L}} \frac{a_{i j U}}{n-(n-1) a_{i j U}}\right)$,

$P(n)$ holds.

Suppose that equation (5.2) holds for $n=m$,

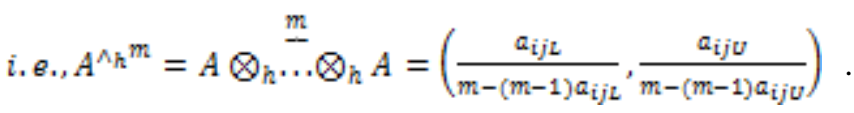

So, when $n=m+1$,

$A^{x_{h} m+1}=\left(\frac{a_{i j L}}{m+1-[(m+1)-1] a_{i j L}}, \frac{a_{i j U}}{m+1-[(m+1)-1] a_{i j V}}\right)$

$A^{N_{h}{ }^{n}}=A \bigotimes_{h} \stackrel{n}{n} \bigotimes_{h} A=\left(\frac{a_{i j L}}{n-(n-1) a_{i j L}}, \frac{a_{i j U}}{n-(n-1) a_{i j U}}\right), \quad$ also holds. 
Using the induction hypothesis that $\mathrm{P}(\mathrm{n})$ holds for any positive integer $n$.

Property 5.3 For any two IVFMs $A$ and $B$ of same size, then positive integer $n$.

$$
\begin{aligned}
& \text { (i) }\left(n_{1 \cdot h} A\right) \bigoplus_{h}\left(n_{2 \cdot h} A\right)=\left(n_{1}+n_{2}\right) \cdot{ }_{h} A, \\
& \text { (ii) }\left(n_{\cdot h} A\right) \bigoplus_{h}\left(n_{\cdot h} B\right)=n_{\cdot h}\left(A \oplus_{h} B\right), \\
& \text { (iii) } A^{\wedge_{h} n_{1}} \bigotimes_{h} A^{\wedge_{h} n_{2}}=A^{\wedge_{h}\left(n_{1}+n_{2}\right)}, \\
& \text { (iv) } A^{\wedge_{h} n} \bigotimes_{h} B^{\wedge_{h} n}=\left(A \bigotimes_{h} B\right)^{\wedge_{h} n}, \\
& \text { (v) } n_{2 \cdot{ }_{h}}\left(n_{1 \cdot{ }_{h}} A\right)=\left(n_{1} n_{2}\right) \cdot{ }_{h} A, \\
& \text { (vi) }\left(A^{\wedge_{h} n_{1}}\right)^{\wedge_{h} n_{2}}=A^{\wedge_{h}\left(n_{1} n_{2}\right)} .
\end{aligned}
$$

Proof. The proof is follows from Theorem 5.1, $5.2 \&$ Definition 3.1.

Property 5.4 For any two IVFMs $A$ and $B$ of same size, then positive integer $\mathrm{n}$.

$$
\begin{aligned}
& (\text { i }) n_{\cdot_{h}}(A \wedge B)=\left(n_{\cdot_{h}} A\right) \wedge\left(n_{\cdot_{h}} B\right), \\
& (\text { ii }) n_{\cdot_{h}}(A \vee B)=\left(n_{\cdot_{h}} A\right) \vee\left(n_{\cdot_{h}} B\right), \\
& (\text { iii })(A \wedge B)^{\wedge_{h} n}=A^{\wedge_{h} n} \wedge B^{\wedge_{h} n}, \\
& \text { (iv) }(A \vee B)^{\wedge_{h} n}=A^{\wedge_{h} n} \vee B^{\wedge_{h} n}
\end{aligned}
$$

Proof. The proof is follows from Theorem 5.1, 5.2 \& Definition 2.4.

\section{CONCLUSION AND FUTURE SCOPE}

The work has expanded the Hamacher operations results under an interval-valued fuzzy matrices. In this paper, we have developed the Hamacher operations of IVFMs and researched their algebraic properties. We likewise demonstrated that the arrangement of all IVFMs concerning Hamacher sum and Hamacher product frames a commutative monoid. An investigation of the mathematical structure of IVFMs as for Hamacher activities gives us a profound knowledge into the applications. At that point, De Morgan's laws are checked. Besides, we developed Hamacher scalar multiplication and Hamacher exponentiation operations an IVFMs and examined their arithmetical properties. we may apply these activities in the field of various zones, for instance, dynamic choice and accord, business and advertising the board, structure, designing and assembling, data innovation and systems administration applications, HR the executives, military applications, vitality the executives, geographic data framework applications, and so forth. It is worth to call attention to that the proposed Hamacher operations over IVFMs will be applied to aggregating interval-valued fuzzy information in the future.

\section{ACKNOWLEDGMENT}

The author would like to thank the referee for improving the readability of the paper.

\section{REFERENCES}

[1] H. Hamacher, Uber logische verknunpfungenn unssharfer Aussagen udderen Zugenhorige Bewertungsfunktione. In:Trappl, Riccardi (eds) Progress in Cybernatics and systems research, 3, 276-288. Hemisphere, Washington.1978.

[2] K.H. Kim, F.W. Roush, "Generalized Fuzzy matrices", Fuzzy Sets and Systems, Vol. 4, Issue.3, pp. 293-315, 1980.

[3] A.R. Meenakshi, M. Kaliraja, "Regular Interval Valued Fuzzy Matrices”.,Advances in Fuzzzy Mathematics, Vol. 5, Issue. 1, pp. 7-15, 2010.

[4] S. Mondal, M. Pal, "Rank of interval-valued fuzzy matrices", Afrika Matematika, Vol. 27, pp. 97-114, 2016.

[5] M. Pal, "Interval-valued Fuzzy Matrices with Interval-valued Fuzzy Rows and Columns", Fuzzy Information and Engineering, Vol. 7, Issue. 3, pp. 335-368, 2015.

[6] A. Pal, and M. Pal, "Some Results on Interval-Valued Fuzzy Matrices". In Proceedings of the 1st International Conference on E-Business Intelligence (ICEBI2010). Atlantis Press. 2010.

[7] M.Z. Ragab, and E.G. Emam, “ The determinant and adjoint of a square fuzzy matrix", Fuzzy Sets and Systems, Vol. 61, Issue.3, pp. 297-307, 1994.

[8] M.Z. Ragab, and E.G. Emam, "On the min-max composition of fuzzy matrices", Fuzzy Sets and Systems, Vol. 75, Issue.1, pp.8392, 1995.

[9] A.K. Shyamal, and M. Pal, "Interval Valued Fuzzy Matrices". Journal of Fuzzy Mathematics, Vol. 14, Issue. 3, pp. 582 592, 2006.

[10] I. Silambarasan, and S. Sriram, "Hamacher sum and Hamacher product of Fuzzy Matrices". International Journal of Fuzzy Mathematical Archive, Vol. 13, Issue. 2, pp. 191-198, 2017.

[11] I.Silambarasan and S.Sriram, "Bounded Sum and Bounded Product of Fuzzy Matrices", Annals of Pure and Applied Mathematics, Vol. 14, Issue. 3, pp. 513-523, 2017.

[12] I.Silambarasan, S. Sriram. "Hamacher Operations of Intuitionistic Fuzzy Matrices". Annals of Pure and Applied Mathematics, Vol. 16, Issue. 1, pp. 81-90, 2018.

[13] I.Silambarasan, "An interval-valued intuitionistic fuzzy matrices based on Hamacher operations". World Scientific News, Vol. 150, pp. 148-161, 2020.

[14]M.G. Thomason, "Convergence of powers of Fuzzy matrix". Journal of Mathematical Analysis and Applications, Vol. 57, Issue. 2, pp. 476-480, 1977. 